\title{
Porphyrin-Heme Biosynthesis in Organotypic Cultures of Mouse Dorsal Root Ganglia

\author{
Effects of Heme and Lead on Porphyrin \\ Synthesis and Peripheral Myelin
}

William O. Whetsell, Jr. University of Tennessee Center for the Health Sciences, Memphis, Tennessee 38163 and Vanderbilt University School of Medicine, Nashville, Tennessee 37232

Shigeru Sassa and Attallah Kappas

The Rockefeller University Hospital, New York, New York 10021

1 bstract. Well-myelinated cultures of mouse dorsal root ganglia incubated for $48 \mathrm{~h}$ with $\delta$-aminolevulinic acid (ALA) showed intense porphyrin fluorescence localized in myelin sheaths but not in axons or neuronal somata. When the cultures were continuously incubated with a high concentration of lead, focal swelling and segmental degeneration of myelin began to develop within 2 wk. Incubation of cultures with ALA after 3 wk of lead treatment revealed markedly decreased porphyrin fluorescence in myelin sheaths compared with untreated controls. After 6 wk of lead treatment, myelin showed severe segmental degeneration. Porphyrin fluorescence from ALA at this time was barely detectable in these cultures. No fluorescence was visible in the demyelinated axons; however, silver-impregnation staining after fixation demonstrated continuity of the axon despite the severe loss of myelin. When cultures were continuously incubated with lead and heme together for $6 \mathrm{wk}$, the segmental demyelination seen in cultures treated with lead alone did not occur. These findings suggest that the lead-induced segmental demyelination in cultured mouse dorsal root ganglia may be due to toxic effects of the metal on the heme biosynthetic pathway in myelinating cells and that exogenous heme may counteract this toxic effect of lead.

\section{Introduction}

Segmental demyelination of peripheral nerves in experimental animals as a consequence of chronic lead toxicity was first de-

Received for publication 5 May 1983 and in revised form 26 March 1984.

J. Clin. Invest.

(C) The American Society for Clinical Investigation, Inc. 0021-9738/84/08/0600/08 $\$ 1.00$

Volume 74, August 1984, 600-607 scribed by Gombault in 1880 (1). Subsequent studies have shown variability in this change in myelin during chronic lead exposure. This variability has been attributed in part to age and species differences in animals and to differences in duration of exposure and doses of lead employed in such studies (2-6). Although there is no conclusive evidence as to whether lead primarily affects neuronal elements, non-neuronal elements, or both, studies dealing with this question (3-5) have suggested that lead produces a primary lesion in Schwann cells and that the neuronal lesions are due, in part, to pathological changes that occur in these supporting cells.

Studies in non-nervous tissues such as hematopoietic cells and liver (7-12) have identified toxic effects of lead on specific enzymes of the heme biosynthetic pathway. Recent studies that used organotypic cultures of dorsal root ganglia derived from the chick $(13,14)$ and the mouse $(15)$ have provided clear evidence for the presence of the porphyrin-heme biosynthetic pathway in this peripheral nervous system tissue. Porphyrin synthetic activity in this tissue system was shown to be localized predominantly in Schwann cells rather than in neuronal cells and was sensitive to the toxic effects of lead (14).

The fact that chronic lead exposure produces damage to Schwann cells (3-5), the demonstration that lead impairs the integrity of the heme biosynthetic pathway (7-12), and the primary localization of the pathway in the supporting elements of dorsal root ganglia (13-15) have led us to study the possible relationship between lead-induced demyelination and impaired porphyrin-heme biosynthesis in this model system.

\section{Methods}

Preparation and treatment of the cultures

Cultures of mouse dorsal root ganglia were selected for this study because of their capacity to produce abundant myelin under normal culture conditions. Cultures were prepared from 16-18-d fetuses. Ganglia were removed under sterile conditions via the dorsal approach. For each culture, two ganglia were placed directly on a collagen-coated glass coverslip carrying $50 \mu \mathrm{l}$ of nutritive medium composed of Eagle's minimal essential medium (60\%), 9-d chick embryo extract (10\%), human placental serum $(30 \%)$, glucose $(6 \mathrm{mg} / \mathrm{ml})$, and nerve growth factor (Collaborative 
Research, Inc., Waltham, MA) $(250 \mathrm{U} / \mathrm{ml})$. The coverslips were then sealed individually into a Maximow chamber (double-coverslip assembly). Subsequently, the cultures received fresh medium twice a week, but no further washing was carried out.

Cultures were examined with bright-field optics and sequentially photographed in the resting state or under experimental conditions. Fixation for light-microscopic staining procedures was carried out by immersing the coverslip directly in the fixative. The coverslip was then taken through standard dehydration and histologic staining procedures for the desired stain, and permanently mounted (culture-side down) on a glass slide.

\section{Experimental procedure}

Treatment with lead. 16 well-myelinated cultures were selected at 21$24 \mathrm{~d}$ for chronic exposure to lead. Lead acetate was added to each culture in medium in a final concentration of $10 \mu \mathrm{M}$. This dosage of lead was shown previously to produce a maximal inhibition of porphyrin formation in organotypic dorsal root ganglion cultures from chick embryos (14). Thereafter, each culture received replenishment of the medium containing the same level of lead twice a week.

Treatment with $\delta$-aminolevulinic acid $(A L A))^{1}$ After exposure to lead acetate for periods of $6 \mathrm{wk}$, nine cultures were incubated with $10 \mathrm{mM}$ ALA for $48 \mathrm{~h}$. The cultures were then examined by dark-field and fluorescence microscopy (primary filter, BG12; secondary filter, BG530; E. Leitz, Inc., Industrial Div., Rockleigh, NJ) and photographed. The individual myelinated fibers in each culture were located first by darkfield microscopy and then examined for porphyrins by fluorescence microscopy, and the fibers were photographed. Sibling control cultures incubated with ALA $(10 \mathrm{mM})$ alone for $48 \mathrm{~h}$ were viewed and photographed by fluorescence microscopy for qualitative comparison of fluorescence.

Treatment with heme. Four well-myelinated cultures were incubated with heme in the medium in a final concentration of $100 \mu \mathrm{M}$ for up to $6 \mathrm{wk}$. As in the lead-exposed cultures, heme was replenished twice a week at the regular intervals. These cultures were sequentially photographed by bright-field optics.

Treatment with heme-plus-lead. 16 well-myelinated cultures were continuously incubated with heme, 0.1-100 $\mu \mathrm{M}$, and lead acetate, 10 $\mu \mathrm{M}$, for periods of up to $6 \mathrm{wk}$. These cultures were sequentially photographed through bright-field optics. Five of the cultures were incubated in $10 \mathrm{mM}$ ALA (after 6 wk lead-plus-heme exposure) and studied by fluorescence microscopy as described above.

Staining procedures. After continuous treatment with lead, heme, or heme-plus-lead for $6 \mathrm{wk}$, cultures were fixed in $1 \%$ osmic acid for $1 \mathrm{~h}$ at room temperature, dehydrated, and stained with Sudan black B for identification of myelin. Other cultures from each experimental group were fixed in $10 \%$ formol/saline and subsequently prepared with silver impregnation, according to a modification of Palmgren's silver-impregnation method used with tissue culture (16), for light-microscopic study of axonal integrity.

\section{Results}

A heavy neuritic outgrowth was visible by light microscopy after 4-6 d of incubation. Individual fibers or fascicles of fibers became progressively prominent for up to $12-14 \mathrm{~d}$, whereas the large ganglion cells showed the characteristic signs of maturation, e.g., development of abundant cytoplasm and a concentric nucleus with prominent nucleolus. At a time between 14 and 18 d, individual nerve fibers within fascicles, or single fibers, dis-

1. Abbreviation used in this paper: ALA, $\delta$-aminolevulinic acid. played evidence of early myelination. At the earliest stages, these fibers bore short segments of distinctive doubly refractile black lines along the axes. As myelination progressed, these short segments became continuous so that dense lines, representing the myelin sheaths, could be observed along the length of the fibers. Periodic interruptions along the course of myelinated fibers produced the typical appearance of nodes of Ranvier. Between the nodes, each segment of myelin showed a flattened Schwann cell nucleus apposed to the outer surface of the myelin sheath. The nodes of Ranvier consistently remained in their original positions along the fibers so that they could serve as landmarks for localization of the individual fibers during chronic experiments (Fig. 1).
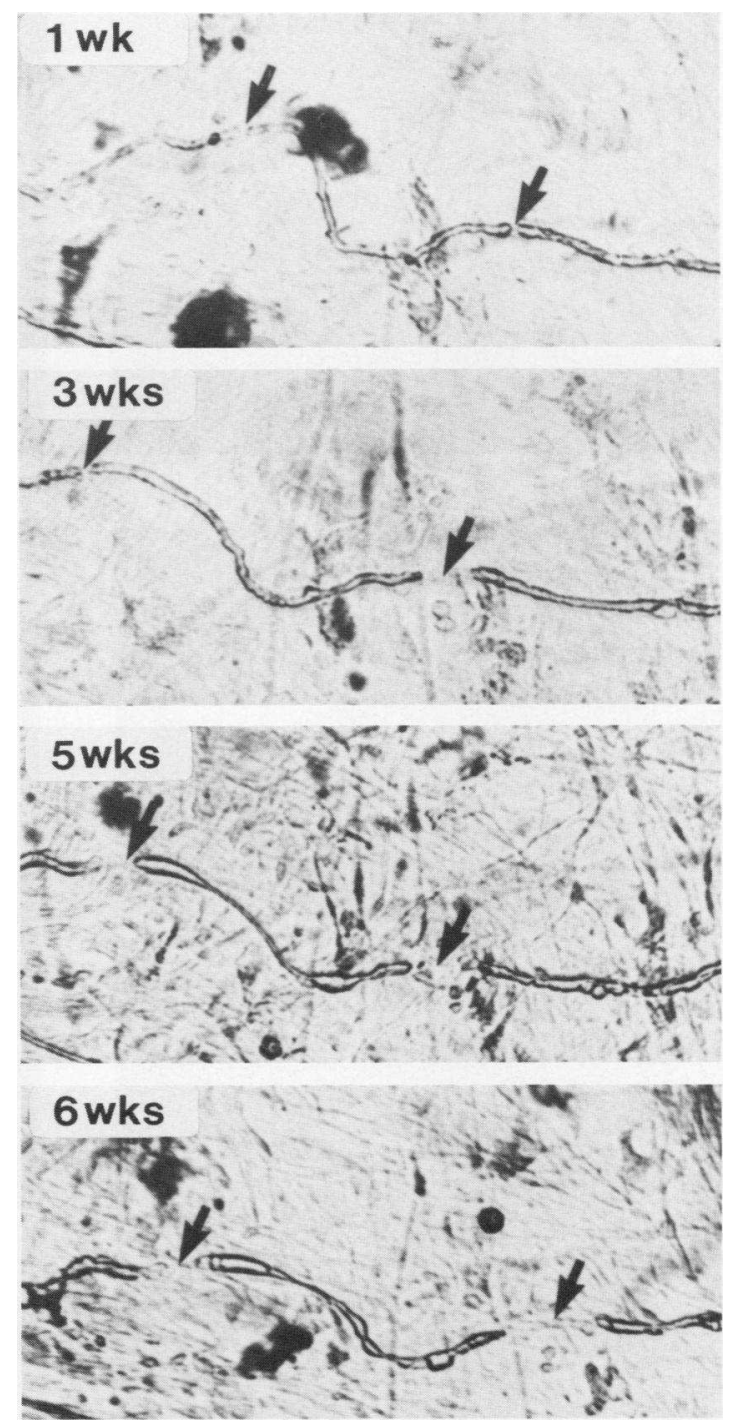

Figure 1. Living myelinated nerve fiber viewed by bright-field light microscopy at $1,3,5$, and 6 wk during continuous exposure to lead $(10 \mu \mathrm{M})$ in the feeding medium. Arrows indicate the same two nodes of Ranvier at each time interval (original magnification of 570). 

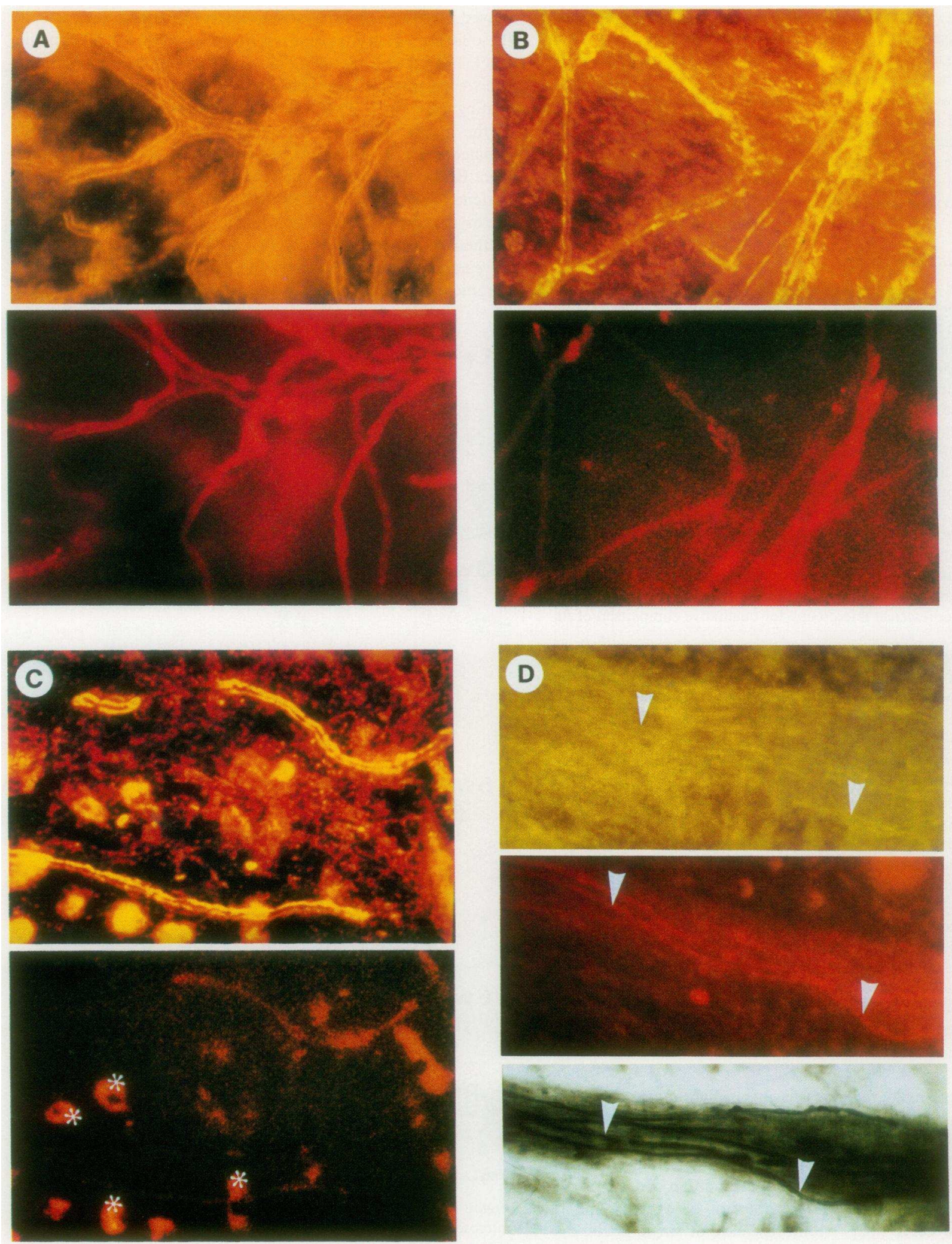
Sequential light-microscopic observations in the experimental cultures showed that by $10-14 \mathrm{~d}$ of lead treatment, individual myelinated nerve fibers showed a consistent though minimal retraction of the ends of adjacent segments of myelin at the nodes of Ranvier. As this early change developed, the nodes of Ranvier became more prominent, the myelin at these points appeared somewhat swollen or bulbous, and axons beneath the retracting myelin became increasingly exposed or bared (Fig. 1). Subsequently focal, then elongated, dilatations occurred along the length of the myelin sheaths. As the loss of myelin progressed, Schwann cell nuclei retained their positions along the axons. At the end of the 6-wk treatment period, the leadtreated cultures showed long gaps in the myelin sheaths, each gap representing an exaggeration of the original node of Ranvier (Fig. 1).

At 3 and $6 \mathrm{wk}$ of lead treatment, cultures were also incubated with ALA ( $10 \mathrm{mM}$ ) for $48 \mathrm{~h}$ and studied and photographed by darkfield and fluorescence microscopy. Compared with control cultures (Fig. $2 A$ ), at 3 wk of lead treatment (Fig. $2 B$ ) porphyrin fluorescence was variable and showed patchy red intensity along the otherwise weakly fluorescent myelin sheath. Neither in control cultures nor in lead-treated cultures was fluorescence observed in the bare axonal regions of the nodes of Ranvier. Porphyrin fluorescence in cultures treated with lead for 6 wk was barely detectable (Fig. $2 C$ ) and could be seen only in the remnants of the myelin sheath along the axons and not in the demyelinated axons themselves.

When the lead-treated cultures were stained for myelin, degeneration of myelin was evidenced by a marked reduction in internodal lengths of the myelin segments, with prominent blebbing and deformation of the remaining segments. The cultures incubated with heme alone for 5 wk showed no visible changes in myelin configuration or in morphology of other structures. At the concentration of heme used (Figs. 2-4), there were no detectable morphological effects on the cultures.

Sequential observations of cultures simultaneously incubated in lead-plus-heme showed that with the lower heme concentrations $(0.1-10 \mu \mathrm{M})$, myelin degeneration indistinguishable from that observed in cultures treated with lead alone was pro- duced. In cultures incubated with the highest dosage of heme $(100 \mu \mathrm{M})$ and with lead, the changes produced in myelin were limited to the appearance of scattered small focal dilatations of the myelin sheaths; no progressive widening of the nodes of Ranvier or disappearance of myelin could be detected in the living cultures (Fig. 3) or in cultures fixed after 6 wk of exposure and stained with Sudan black B (Figs. $2 D$ and $4 C$ ). These findings were confirmed by morphometry (Table I). The latter cultures, however, when incubated with ALA for $48 \mathrm{~h}$, exhibited diminution of porphyrin fluorescence (Fig. $2 \mathrm{D}$ ) similar to that observed in cultures exposed to lead alone. Control cultures incubated with heme alone $(100 \mu \mathrm{M})$ for 6 wk showed no changes in the appearance of myelinated fibers.

Silver-impregnation staining of lead-treated cultures showed that despite the presence of severe retraction and loss of myelin, the axis cylinders could still be shown to retain their original courses and configurations over the collagen surface (Fig. 5). A striking incidental observation with silver-stain preparations was the abundance of small unmyelinated nerve fibers still present after the 6-wk lead treatment period.

\section{Discussion}

The results of the present study support the idea that there is a relationship between lead-induced impairment of porphyrinogenesis in cultured dorsal root ganglia cells and the demyelinating process produced as a consequence of sustained exposure of such cells to this metal. In this study control cultures of mouse dorsal root ganglia incubated with ALA for $48 \mathrm{~h}$ developed a prominent porphyrin fluorescence which was observed almost exclusively in myelin sheaths; no fluorescence was detected in the nerve fibers themselves, whether or not they were myelinated. When cultures were continuously exposed to lead under the conditions described and then incubated with ALA for $48 \mathrm{~h}$, the intensity of the porphyrin fluorescence in myelin sheaths was progressively diminished as the treatment time increased. Simultaneously, the myelin sheaths underwent segmental degeneration characterized first by focal swelling and then shrinkage away from the nodes of Ranvier so that the nodes became increasingly wide. Continuity of demyelinated axons could,
Figure 2. (A) Upper panel: Dark-field photograph of normal (control) myelin in culture; note smooth appearance of myelinated nerve fibers. Lower panel: Fluorescence photograph of the same fibers showing intense porphyrin fluorescence. $(B)$ Upper panel: Dark-field photograph of myelinated fibers after 3 wk exposure to lead $(10 \mu \mathrm{M})$; note beaded irregularities of the fibers, reflecting focal degeneration of myelin. Lower panel: Fluorescence appearance of the same fibers; note moderate diminution and patchy distribution of porphyrin fluorescence along the fibers. $(C)$ Upper panel: Dark-field appearance of the nerve fibers after $6 \mathrm{wk}$ exposure to lead $(10 \mu \mathrm{M})$; note the marked fragmentation of myelin. Lower panel: Fluorescence photograph of the same nerve fibers; note the greatly diminished porphyrin fluorescence in the fibers. Prominently fluorescing cells, which appear to be macrophages (white asterisks) are also seen; such cells are only occasionally observed in control $(A)$ cultures. $(D)$ Top panel: Darkfield appearance of a bundle of myelinated fibers after $6 \mathrm{wk}$ of lead
$(10 \mu \mathrm{M})$-plus-heme $(100 \mu \mathrm{M})$ treatment. White arrows indicate myelinated fibers; note that the myelin appears smooth and continuous as in the control $(A)$ culture. Middle panel: Fluorescence photograph of the same fibers (white arrows) showing diminished porphyrin fluorescence compared with control $(A)$ cultures. Bottom panel: Brightfield photomicrograph of the same fibers (white arrows) after fixation and staining with Sudan black B; note the continuity and preservation of myelin (original magnification in all photographs of 250). The variability of color in all dark-field photographs is related to diffraction of dark-field illumination by the collagen bed and fiber network associated with the myelinated fibers; the variability is greater in some cases because of the increased exposure time necessary to bring out details of the myelinated fibers. The variable intensity of the background in the fluorescence photographs is due to variations in the exposure time (30 to $120 \mathrm{~s}$ ) required to record fluorescence in the fibers. 

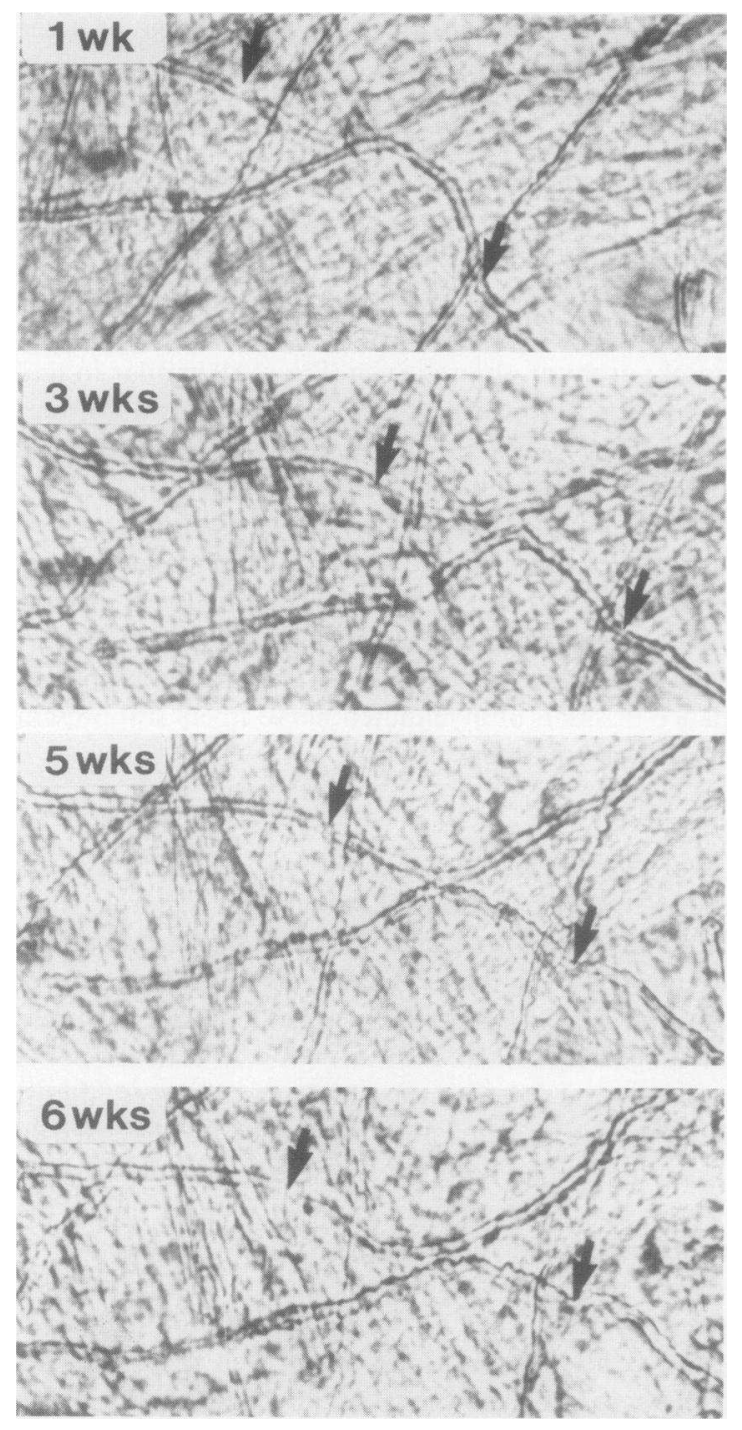

Figure 3. Living myelinated nerve fibers (bright-field optics) at 1, 3, 5 , and $6 \mathrm{wk}$ of continuous exposure to lead $(10 \mu \mathrm{M})$-plus-heme (100 $\mu \mathrm{M})$. Arrows indicate the same two nodes of Ranvier at each interval; note preservation of myelin and normal appearance of the nodes (original magnification of 570).

however, be demonstrated by silver impregnation staining even after severe loss of myelin had occurred. In cultures continuously treated with lead and a high concentration of heme for $6 \mathrm{wk}$, myelin was spared the severe degenerative changes observed in the cultures exposed to lead alone. Porphyrin fluorescence in these lead-plus-heme treated cultures was diminished to an extent equivalent to that seen in lead-treated cultures; however, the myelin sheaths in the lead-plus-heme cultures were well preserved, in contrast to cultures exposed to lead alone. Morpho-

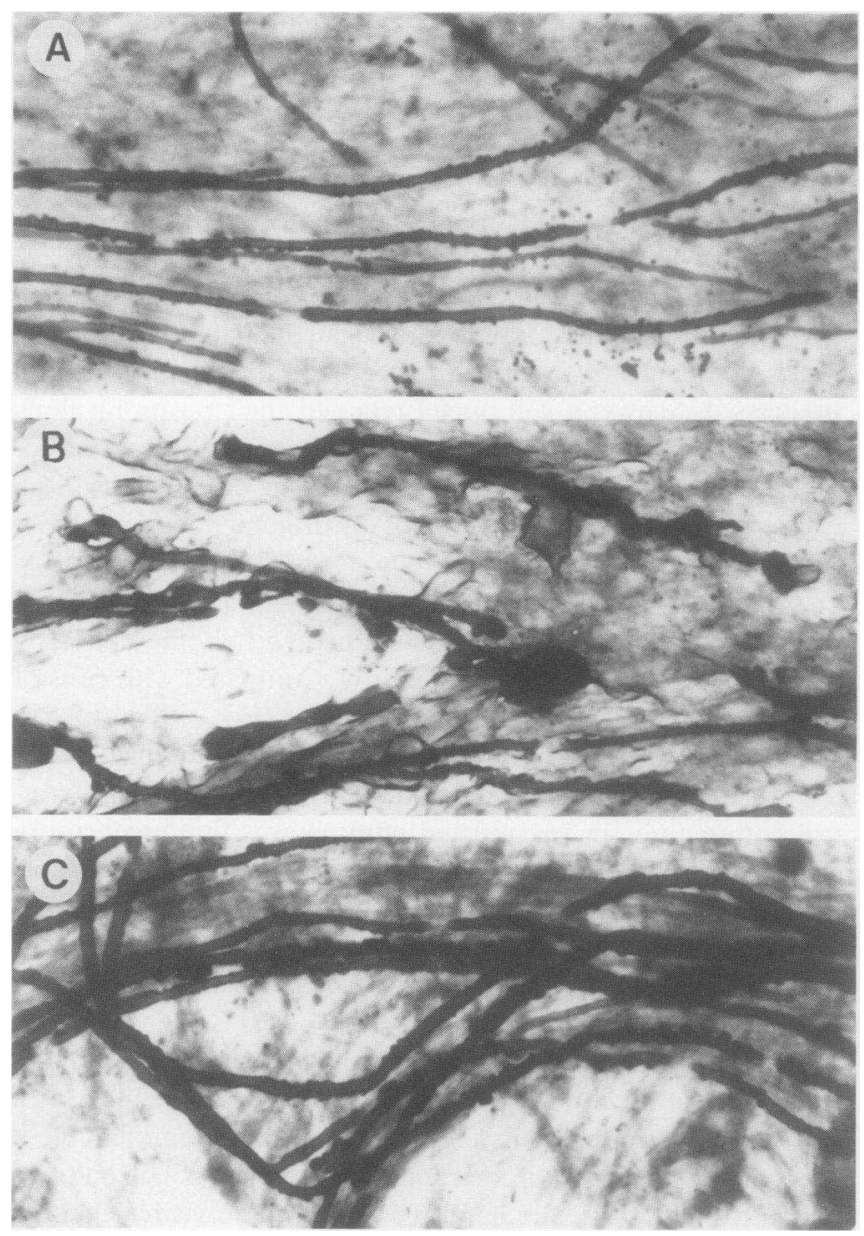

Figure 4. Comparison of the appearance of myelin after fixation and Sudan black B stain; in normal, control culture $(A)$; after 6 wk continuous exposure to lead $(10 \mu \mathrm{M})(B)$; and after 6 wk continuous exposure to lead $(10 \mu \mathrm{M})$-plus-heme $(100 \mu \mathrm{M})(C)$. Note the marked fragmentation of myelin in the lead-treated culture $(B)$ as compared with the well-myelinated fibers in the control $(A)$ or the lead-plusheme-treated culture $(C)$ (original magnification of 650 ).

metric analysis of nerve fibers corroborated the protective effect of heme against lead-induced demyelination (Table I).

The earliest systematic description of pathological findings in experimental lead neuropathy is that of Gombault (1), who observed that during chronic lead exposure, guinea pigs developed a peripheral neuropathy characterized by segmental demyelination but without apparent damage to axons. Subsequent studies have reported both segmental demyelination and Wallerian degeneration, which occur to a variable degree depending on the species and the age of animals and on the dosage and duration of lead treatment (2-6). Although both Wallerian degeneration and segmental demyelination have been described, 
Table I. Morphometric Comparison of Nodes of Ranvier in Lead and Lead-Plus-Heme Treated Cultures

\begin{tabular}{lllllll}
\hline & $0-1 \mathrm{wk}$ & $2 \mathrm{wk}$ & $3 \mathrm{wk}$ & $4 \mathrm{wk}$ & $5 \mathrm{wk}$ & $6 \mathrm{wk}$ \\
\hline $\begin{array}{l}\text { Lead treatment } \\
\begin{array}{l}\text { Lead-plus-heme } \\
\text { treatment }\end{array}\end{array}$ & $1.4 \pm 0.2 \mu \mathrm{m} \mathrm{(4)}$ & $1.4 \pm 0.3 \mu \mathrm{m} \mathrm{(4)}$ & $2.6 \pm 1.1 \mu \mathrm{m}(6)$ & $3.4 \pm 2.4 \mu \mathrm{m}(3)$ & $4.9 \pm 1.5 \mu \mathrm{m}(5)$ & $5.3 \pm 0.6 \mu \mathrm{m}(5)$ \\
& $1.4 \pm 0.1 \mu \mathrm{m}(4)$ & $1.5 \pm 0.1 \mu \mathrm{m} \mathrm{(4)}$ & $1.7 \pm 0.2 \mu \mathrm{m} \mathrm{(4)}$ & $1.7 \pm 0.2 \mu \mathrm{m}(4)$ & $1.7 \pm 0.2 \mu \mathrm{m}(4)$ & $2.1 \pm 0.5 \mu \mathrm{m}(4)$
\end{tabular}

Cultures were treated with lead $(10 \mu \mathrm{M})$ alone or lead-plus-heme $(100 \mu \mathrm{M})$ for up to $6 \mathrm{wk}$, and the widths of the nodes of Ranvier $(n=3-6)$ were determined in the cultures and expressed as the mean \pm SD. Numbers of nodes measured are indicated in parentheses. No changes in the nodes of Ranvier were noted in cultures incubated in heme alone for 6 wk.

there are indications that damage to Schwann cells by lead may represent the primary lesion responsible for demyelination as well as for axonal degeneration. Schlaepfer (3) has suggested that the combination of sensory neuronal degeneration and segmental demyelination observed in rats exposed to lead for periods of 3 to 18 mo could be explained by a metabolic lesion in Schwann cells, including satellite cells.

Biochemical mechanisms involved in lead toxicity in the nervous system have not been fully elucidated. Lead neuropathy may result from the effects of the metal on different elements of the nervous system including vessels, Schwann cells, stromal cells, and nerve cells (6). Several hypotheses concerning possible biochemical mechanisms for lead toxicity in the central nervous system in experimental models have been proposed (17-19). However, there has been less focus on the possible mechanism of the demyelination produced by lead in the peripheral nervous system $(2-6,20)$. Studies dealing with this aspect of lead toxicity have suggested either that a lead-induced disturbance of calcium transport may cause swelling and damage to Schwann cells (20)

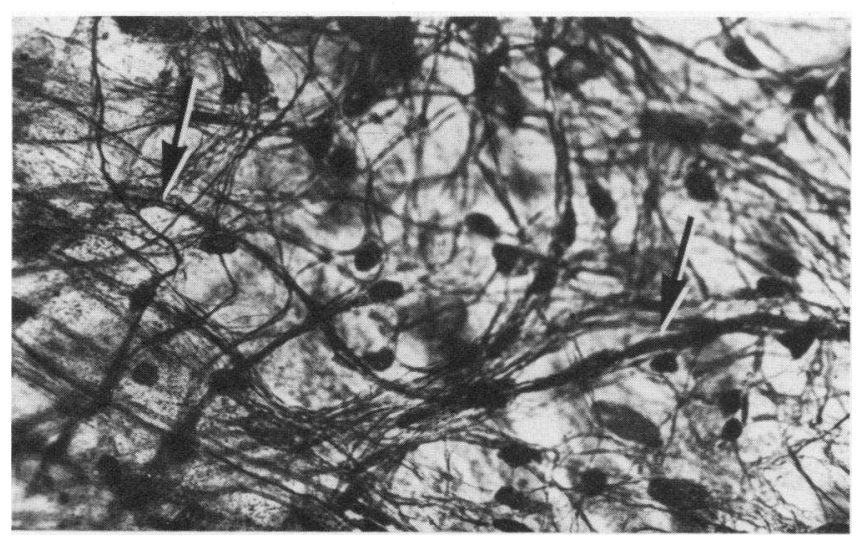

Figure 5. Photomicrograph showing the same field as that shown in Fig. 1 (6 wk) after fixation and silver staining. The arrows are in the same position along the same fiber displayed in Fig. 1. Despite the extensive damage to myelin shown in Fig. 1, there are abundant wellpreserved nerve fibers demonstrated by the silver stain (original magnification of 570). or that the metal may inhibit the porphyrin-heme biosynthetic pathway in non-neuronal supporting elements, specifically those cells responsible for myelin formation (13-15).

The studies of Klüver $(21,22)$ and more recently of Asbury (23) have indicated the presence of a porphyrin biosynthetic pathway in the nervous system (13-15). The possible relationship between the toxic effects of lead on this pathway and on the myelination of nerve fibers in culture have not been previously examined. A major difficulty in conducting such studies had been the unavailability of living, undisturbed, morphologically intact, and biochemically active nervous tissues. The availability of techniques for culturing nervous system tissue organotypically $(24,25)$ so that the various components retain their morphologic as well as biochemical cell-to-cell relationships in vitro now permits examination of the activity of the porphyrin-heme pathway and the effect on this pathway of the prototype environmental toxin, lead. Previous studies in cultures of avian and mammalian peripheral nervous system tissues have demonstrated an intrinsic porphyrin-heme biosynthetic pathway in such tissues and suggested that the pathway is principally localized to non-neuronal elements, specifically Schwann cells and other supporting cells (13-15). These cells, elements of which are critically involved in myelin formation, have been shown to be able to synthesize at least six porphyrin intermediates of the heme biosynthetic pathway when incubated with a precursor, ALA (14).

The myelin degeneration and concomitant diminution of induced porphyrin fluorescence observed in the ganglion cultures during chronic lead exposure indicate that this metal may produce local inhibition of the porphyrin-heme biosynthetic sequence. As a consequence of this inhibition, porphyrin formation from the precursor, ALA, is reduced, and the conversion of porphyrins to heme is probably also inhibited. Evidence on this point is circumstantial in studies that use nerve cell cultures but is consistent with the effects of lead on ferrochelatase activity in other tissues treated with the metal $(5,14)$. If heme is essential to the metabolic capacity of Schwann cells to maintain or form myelin, then deprivation of heme through such an inhibitory mechanism could be involved in the degenerative changes that occur in the peripheral nervous system in lead toxicity. Segmental demyelination may represent an early visible sign of Schwann 
cell damage as a consequence of lead inhibition of heme synthesis. This idea is consistent with the observation in this study that exogenously supplied heme was able to exert a protective effect against lead-induced myelin degeneration in the cultured nerve cells. The mechanism of this protective effect of heme on myelin preservation is not known. It is conceivable that when cellular heme synthesis is inhibited by lead, an exogenous supply of heme may circumvent the lead effect, thus preserving the functional integrity of Schwann cells that sustain the myelin sheath. It is of interest in this regard that exogenous heme may reverse some of the abnormalities in patients with lead poisoning (26).

It should be emphasized that a high concentration of exogenous heme was required to counteract the toxic effect of lead on nerve myelin formation in this study. High concentrations $(50-100 \mu \mathrm{M})$ of heme are also required for stimulation of protein synthesis in in vitro cell-free systems (27); stimulation of neuronal differentiation of mouse neuroblastoma cells (28); erythroid differentiation of mouse (29) and human (30) erythroleukemia cells; formation of erythroid colonies in normal mouse bone marrow cultures (31); differentiation of 3T3 fibroblasts into adipocytes (32); and stimulation of cell growth of cultured fibroblasts $(33,34)$. Thus the pharmacological dosage of heme required to counteract the toxic effect of lead on myelin formation in cultured ganglion cells is consistent with the amounts of this compound required to elicit a wide variety of biochemical, developmental, and morphological changes in other cellular systems.

\section{Acknowledgments}

The authors thank Ms. Julia Shen, Ms. Westelle Sweeney, and Ms. Karen Anderson for their excellent technical assistance.

This research was supported by U. S. Public Health Service grants ES- 0241 and ES-01055 and by gifts from the Ogden Corporation and the Renfield Foundation.

\section{References}

1. Gombault, A. 1880. Contribution à l'étude anatomique de nebrite parenchymateuse subaigue et chronique. Arch. Neurol. 1:11-38.

2. Fullerton, P. M. 1966. Chronic peripheral neuropathy produced by lead poisoning in guinea-pigs. J. Neuropath. Exp. Neurol. 25:214236.

3. Schlaepfer, W. A. 1969. Experimental lead neuropathy: a disease of the supporting cells in the peripheral nervous system. J. Neuropath. Exp. Neurol. 28:401-417.

4. Lampert, P. W., and S. S. Schochet, Jr. 1968. Demyelination and remyelination in lead neuropathy. J. Neuropath. Exp. Neurol. 27:527545.

5. Ohnishi, A., K. Schilling, W. S. Brimijoin, E. H. Lambert, V. F. Fairbanks, and P. J. Dyck. 1977. Lead Neuropathy. I. Morphometry, nerve conduction and choline acetyltransferase: new finding of endoneurial edema associated with segmental demyelination. J. Neuropath. Exp. Neurol. 36:499-518.

6. Krigman, M. R., T. W. Bouldin, and P. Mushak. 1980. Lead. In
Experimental and Clinical Neurotoxicology. P. C. Spencer and H. H. Schaumber, editors. Williams and Wilkins, Baltimore. 490-507.

7. Sassa, S., J. L. Granick, S. Granick, A. Kappas, and R. D. Levere. 1973. Studies in lead poisoning. I. Micro analysis of erythrocyte protoporphyrin levels by spectrofluorometry in the detection of chronic lead intoxication in the subclinical range. Biochem. Med. 8:135-148.

8. Granick, J. L., S. Sassa, S. Granick, R. D. Levere, and A. Kappas. 1973. Studies in lead poisoning. II. Correlation between the ratio of activated to inactivated delta aminolevulinic acid dehydratase of whole blood and the blood lead level. Biochem. Med. 8:149-159.

9. Buchet, J. P., H. Roels, G. Hubermont, and R. Lauwerys. 1976. Effect of lead on some parameters of the heme biosynthetic pathway in rat tissues in vivo. Toxicology. 6:21-34.

10. Gibson, S. L. M., and A. Goldberg. 1970. Defects in haem synthesis in mammalian tissues in experimental lead poisoning and experimental porphyria. Clin. Sci. 38:63-72.

11. Chisolm, J. J., Jr., M. B. Barrett, and H. V. Harrison. 1975. Indicators of internal dose of lead in relation to derangement in heme synthesis. Johns Hopkins Med. J. 137:6-12.

12. Sassa, S. 1978. Toxic effects of lead, with particular reference to porphyrin and heme metabolism. In Handbook of Experimental Pharmacology. Heme and Hemoproteins. F. DeMatteis and W. N. Aldrige, editors. Springer Verlag, Berlin. 44:333-371.

13. Whetsell, W. O., Jr., S. Sassa, D. Bickers, and A. Kappas. 1978. Studies on porphyrin-heme biosynthesis in organotypic cultures of chick dorsal root ganglion. I. Observations on neuronal and non-neuronal elements. J. Neuropath. Exp. Neurol. 37:497-507.

14. Sassa, S., W. O. Whetsell, Jr., and A. Kappas. 1979. Studies on porphyrin heme biosynthesis in organotypic cultures of chick dorsal root ganglia. II. The effect of lead. Environ. Res. 19:415-426.

15. Whetsell, W. O., Jr., S. Sassa, and A. Kappas. 1979. Studies on effects of chronic lead exposure upon porphyrin biosynthesis and myelin in cultures of mouse dorsal root ganglia (DRG). J. Neuropath. Exp. Neurol. 38:348. (Abstr.)

16. Ecob-Johnston, M. S., J. Schwartz, T. S. Elizan, and W. O. Whetsell, Jr. 1978. Herpes simplex virus types 1 and 2 in organotypic cultures of mouse central and peripheral nervous system. I. Light microscopic observations of myelin degeneration. J. Neuropath. Exp. Neurol. 37:518-520.

17. Nathanson, J. A., and F. E. Bloom. 1976. Heavy metals and adenosine cyclic $3^{\prime}, 5^{\prime}$-monophosphate metabolism: possible relevance to heavy metal toxicity. Mol. Pharmacol. 12:390-398.

18. Muller, W. E., and S. H. Snyder. 1977. Delta aminolevulinic acid: influence of synaptic GABA receptor binding may explain CNS symptoms of porphyria. Ann. Neurol. 2:340-342.

19. Silbergeld, E. K., and H. S. Adler. 1978. Subcellular mechanisms of lead neurotoxicity. Brain Res. 148:451-467.

20. Camer, W. 1980. Toxic demyelination: biochemical studies and hypothetical mechanisms. In Experimental and Clinical Neurotoxicity. P. C. Spencer and H. H. Schaumberg, editors. Williams and Wilkins, Baltimore. 239-256.

21. Klüver, H. 1944. Porphyrins, the nervous system and behavior. J. Psych. 17:209-277.

22. Klüver, H. 1944. On naturally occurring porphyrins in the central nervous system. Science (Wash. DC). 99:482-484.

23. Asbury, A. K., R. L. Sidman, and M. K. Wolf. 1966. Druginduced porphyrin accumulation in the nervous system. Neurology. 16:320. (Abstr.)

24. Murray, M. R. 1971. Nervous tissues isolated in culture. In 
Handbook of Neurochemistry. A. Lajtha, editor. Plenum Press, New York. 5A:373-438.

25. Bunge, M. D., R. P. Bunge, E. R. Peterson, and M. R. Murray. 1967. A light and electron microscopic study of long term organized cultures of rat dorsal root ganglia. J. Cell Biol. 32:439-466.

26. Silbergeld, E. K., and J. M. Lamon. 1982. Effects of altered porphyrin synthesis on brain neurochemistry. Neurobehav. Toxicol. Teratol. 4:635-642.

27. London, I. M., V. Ernst, R. Fagard, A. Leroux, D. H. Levin, and R. Petryshyn. 1981. Regulation of protein synthesis by phosphorylation and heme. Cold Spring Harbor Conf. Cell Proliferation. 8:941957.

28. Ishii, D. N., and G. M. Maniatis. 1978. Hemin promotes rapid neurite growth in cultured mouse neuroblastoma cells. Nature (Lond.). 274:373-374.

29. Granick, J. L., and S. Sassa. 1978. Hemin control of heme biosynthesis in mouse Friend virus-transformed erythroleukemia cells in culture. J. Biol. Chem. 253:5402-5406.

30. Benz, E. J., M. J. Murnane, B. L. Tonkonow, B. W. Borman, E. M. Mazur, C. Cavallesco, T. Jenko, E. L. Snyder, B. G. Forget, and R. Hoffman. 1980. Embryonic fetal erythroid characteristics of human leukemic cell line. Proc. Natl. Acad. Sci. USA. 77:3509-3513.

31. Porter, P. N., R. H. Meints, and K. Mesner. 1979. Enhancement of erythroid colony growth in culture by hemin. Exp. Hematol. 7:1116.

32. Chen, J.-J., and I. M. London. 1981. Hemin enhances the differentiation of mouse 3T3 cells to adipocytes. Cell. 26:117-122.

33. Verger, C. 1979. Proliferation and morphology of chick embryo cells cultured in the presence of horse serum and hemoglobin. In Vitro. 15:587-592.

34.Verger, C., S. Sassa, and A. Kappas. 1983. Growth-promoting effects of iron- and cobalt-protoporphyrins on cultured embryonic cells. J. Cell Physiol. 116:135-141. 